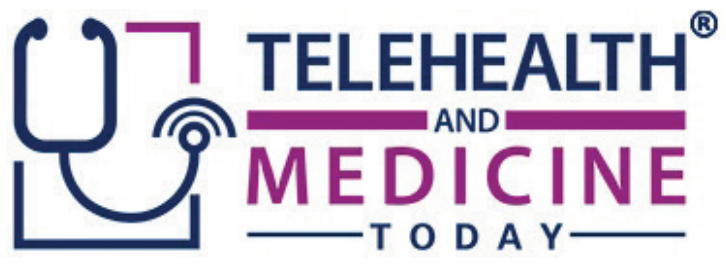

\title{
Space Medicine: The Ultimate in Remote Healthcare
}

\section{K. Ganapathy}

Affiliation: Apollo Telemedicine Networking Foundation \& Apollo Telehealth Services, Chennai, India

Corresponding Author: Prof. K. Ganapathy, Email: drganapathy@apollohospitals.com

Keywords: Space Medicine, Remote Healthcare, Telehealth in Outer Space

Section: Opinions, Perspectives, and Commentary on a current trend or issue impacting the sector

The term "Remote Healthcare" (RHC) clearly

defines what is implied by telehealth or

telemedicine. The latter terms clearly indicate

delivering healthcare at a distance using

information and communication technology.

Distance today has become meaningless - at

least on earth! Geography has indeed become

History. The ultimate in providing RHC would

be to monitor continuously in almost real time,

various health parameters of those in outer

space-staying in low earth orbit at present.

Eventually, this would extend to the lunar

surface, deep space, and one day perhaps, even

on the red planet, Mars. This overview discusses some of these challenges.

$\mathrm{S}$ pace travel, once considered esoteric and part of science fiction, is becoming a reality. From 1961 to June 2018, 561 individuals from 40 countries have travelled so far to outer space, on over 1,230 spaceflights, accounting for 46,947 person-days in space. The National Aeronautics and Space Administration's
(NASA) Johnson Space Center (Figure 1) has served as a hub of human spaceflight activity for more than half a century. The flight surgeons at the Center do a commendable job in providing RHC in all manned space missions.

Seventeen nonfatal yet severe medical emergencies have been documented during spaceflights. The Russian cosmonaut Valeri Polyakov spent 438 days onboard the Mir space station. ${ }^{1}$ Several private organizations have already announced plans to commence space tourism. ${ }^{2}$ The presence of humans in space missions poses many challenges to both the engineering system designers and medical professionals to develop systems and procedures that could ensure the safety and comfort of the crew. ${ }^{3}$ Astronauts selected are normally in excellent health - physically and psychologically. However, unanticipated acute or chronic illnesses requiring early or immediate diagnosis may still occur. Emergencies might include minor and major accidents, burns, injuries, trauma, heart attacks, strokes, emboli, and infections. 


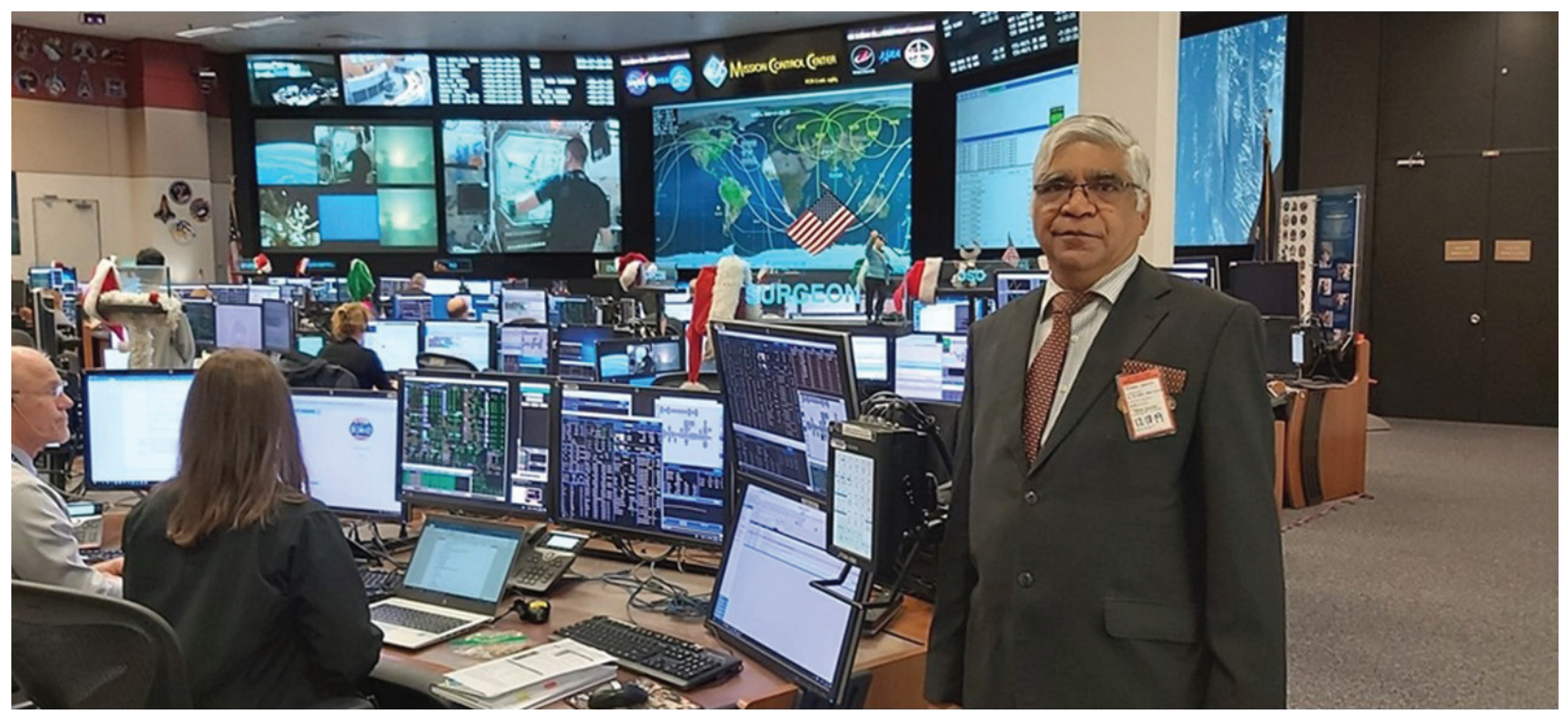

Figure 1. The author at NASA's Johnson Space Center, Houston.

\section{SPECIFIC HEALTH HAZARDS IN OUTER SPACE}

As extra-terrestrial space is a hostile environment, ${ }^{4}$ spacecraft and equipment must protect astronauts within and during extravehicular activities (EVA). The cloistered internal space in the spacecraft itself is potentially intimidating, physically and psychologically. Long missions cause prolonged isolation from family and daily activities of living on Earth. This itself might lead to anxiety and depression. This is in addition to the effects of microgravity, radiation, and disturbance in circadian rhythms.

\section{REMOTE HEALTHCARE FOR ASTRONAUTS}

NASA has identified many hazards that might affect human health in space. Distance adds substantially to any risk but especially during emergencies. Distances are in the realm of 420 $\mathrm{km}$ ( $\sim 260$ miles) above the earth and 380,000 km ( 236,000 miles) beyond the earth. For example, when humans will go to Mars in another 10-15 years, healthcare must be provided 57.6 million km (35.8 million miles) away (the nearest possible distance from earth). Remote physical examination, onboard lab tests, and medical imaging will be necessary.

Modified point of care diagnostics and handheld ultrasound scanners could be useful for diagnosing abscesses, clots in blood vessels, and calculi in kidneys or gallbladder. An obstructive jugular vein thrombosis developed in an astronaut when the astronaut was 2 months into a 6-month space mission. This was managed from Earth. The limited onboard supply of medicine required carefully meting out the dose until a fresh cargo shipment arrived from Earth. ${ }^{5}$

At present, general anesthesia administration or performing conventional surgical procedures in microgravity is not possible. Administering basic life support in microgravity is available. Minor surgical procedures, such as vessel and wound closures, have been performed only during research studies in space on small animals. For minor surgical interventions, training of the crew is necessary. 
Lightweight medical robots could enhance the medical skills of trained astronauts. During the mission, new healthcare information can be uploaded in real time onto onboard computers. This medical record could be stored within a digital "super-doc" capable of diagnosis and therapeutic triage. Unobtrusive onboard remote sensing devices, which continuously monitor vital signs, blood cell levels, blood biochemistry, and behavioral changes, could send alerts to the rest of the crew, ground personnel, and the onboard "super-doc" when deviations from baseline health are detected.

Crews on the International Space Station (ISS) and previous human spaceflight missions were linked to the ground through a network permitting establishment of telemedicine communication. ${ }^{6}$ Long-duration stays on the ISS are accompanied by significant effects on the crew's cardiovascular, vestibular, and musculoskeletal systems. Bone loss and muscle atrophy occur at 1 to $3 \%$ and $5 \%$ monthly, respectively. Oxygen consumption is reduced by about $25 \%$ after a few weeks in space. The medical system on the ISS is adequate to address a wide variety of the needs of the crews. Each mission has a designated crew medical officer (CMO). Medical kits have necessary medications, and there are limited diagnostic tools.

\section{TELECOMMUNICATIONS RELEVANT TO EXTRATERRESTRIAL REMOTE HEALTHCARE}

Ground stations can communicate with low earth orbit satellites only when the satellite is in their visible region. Contact communication time could take 5-15 minutes, with an average frequency of 6-8 times daily. A full orbit (24 hours earth time) in outer space takes 90-110 minutes. Every satellite carries special instruments.
The satellite coverage area is defined as a region of the Earth, where the satellite is seen at a minimum predefined elevation angle. Reduced time delay in communication is mandatory for "real-time" healthcare. Communication between Earth and Mars will be characterized by significant time delays varying from 3 to 22 minutes one way.

Mars-bound crews must have a complex medical system onboard to address the medical needs. Continuous healthcare monitoring by ground-based medical personnel presupposes a secure communication link. The Indian Space Research Organisation (ISRO) will be launching two dedicated communication satellites-Indian Data Relay Satellite System (IDRSS) - to ensure that the vyomanauts (Indian term for astronauts) remain in constant touch with ISRO scientists throughout the mission. This will also facilitate providing RHC. The IDRSS satellites, one opposite to each half of earth in geosynchronous equatorial orbit, can see about $80 \%$ of the area where Indian remote sensing satellites are orbiting, hence enhancing the visibility range and data transfer rates of satellites. Satellites will hereby reduce the dependency on ground stations. Implementation of the system will help real-time tracking of Gaganyaan, India's first Human Space Flight Mission.

\section{CONCLUSIONS}

For a neurosurgeon trained in the Before Computers (BC) era, in what was then referred to as a third-world country, to actually witness RHC preparations for India's first vyomanauts who will be in low earth orbit in just 22 months from now clearly demonstrates that the future is always ahead of schedule. It was Mark Twain who once remarked, "The future ain't (aka 'is not') what it used to be." 
Many of us fear the future and cling desperately to the present, not realizing the fact that we have already become the past. We need to be futureready. I have no doubt that preparations for extraterrestrial RHC, which will soon commence in India - the ultimate in telehealth - will eventually result in tremendous advances for our fellow brethren confined to earth. For onesixth of humanity, the proposed Human Space Flight Mission from India will indeed be an extraordinary challenge. Undoubtedly, this will result in an exponential growth of RHC on earth! After all, at least 1,770 innovations have been attributed as spin-offs from work done by NASA. ${ }^{7}$

\section{Acknowledgments: None.}

Conflict of Interests: The author declares no potential conflicts of interest.

Contributors: The author wrote all sections of this article.

Funding statement: No funding was received from any source for preparing this article for publication.

\section{REFERENCES}

1. Doarn CR, Polk JD, Shepanek M. Health challenges including behavioral problems in long-duration spaceflight. Neurol India. 2019;67(Suppl S2):190-5. https://doi. org/10.4103/0028-3886.259116

2. Ganapathy K, da Rosa M, Russomano T. Neurological changes in outer space. Neurol India. 2019;67:37-43.
3. Nair SU. Challenges to human spaceflight program: The emerging role of bioastronautics. Neurol India. 2019;67(Suppl S2):167-8. https://doi. org/10.4103/0028-3886.259119

4. Kandarpa K, Schneider V, Ganapathy K. Human health during space travel: An overview. Neurol India. 2019;67(Suppl S2):176-81. https://doi.org/10.4103/00283886.259123

5. Auñón-Chancellor SM, Pattarini JM, Moll S, Sargsyan A. Venous thrombosis during spaceflight. $N$ Engl $J$ Med. 2020;382:89-90. https://doi.org/10.1056/ NEJMc1905875

6. Doarn CR, Nicogossian AE, Merrell RC. Applications of telemedicine in the United States space program. Telemed $J$. 1998;4:19-30. https://doi.org/10.1089/ tmj.1.1998.4.19

7. Ganapathy K. Extra-terrestrial neurosciences. Neurol India. 2019;67(Suppl S2):165-6. https://doi.org/10.4103/00283886.259118

Copyright Ownership: This is an open access article distributed in accordance with the Creative Commons Attribution Non Commercial (CC BY-NC 4.0) license, which permits others to distribute, adapt, enhance this work non-commercially, and license their derivative works on different terms, provided the original work is properly cited and the use is noncommercial. See: http://creativecommons. org/licenses/by-nc/4.0. 